\title{
Flexible Tardiness Bounds for Sporadic Real-Time Task Systems on Multiprocessors *
}

\author{
UmaMaheswari C. Devi and James H. Anderson \\ Department of Computer Science, The University of North Carolina at Chapel Hill
}

\begin{abstract}
The earliest-deadline-first (EDF) scheduling of a sporadic real-time task system on a multiprocessor may require that the total utilization of the task system, $U_{\text {sum }}$, not exceed $(m+1) / 2$ on $m$ processors if every deadline needs to be met. In recent work, we considered the alleviation of this underutilization for task systems that can tolerate deadline misses by bounded amounts (i.e., bounded tardiness). We showed that if $U_{\text {sum }} \leq m$ and tasks are not pinned to processors, then the tardiness of each task is bounded under both preemptive and non-preemptive EDF. However, the tardiness bounds derived are applicable to every task in the task system, i.e., any task may incur maximum tardiness. In this paper, we consider supporting tasks whose tolerances to tardiness are less than that known to be possible under EDF. We propose a new scheduling policy, called EDF-hl, which is a variant of EDF, and show that under EDF-hl, any tardiness, including zero tardiness, can be ensured for a limited number of privileged tasks, and that bounded tardiness can be guaranteed to the remaining tasks if their utilizations are restricted. EDF-hl reduces to EDF in the absence of privileged tasks. The tardiness bound that we derive is a function of $U_{\text {sum }}$, in addition to individual task parameters. Hence, tardiness for all tasks can be lowered by lowering $U_{\text {sum. }}$. A simulation-based evaluation of the tardiness bounds that are possible is provided.
\end{abstract}

\section{Introduction}

A real-time system has to meet certain timing constraints to be correct. Such timing constraints are typically specified as deadlines for tasks. Tasks in a real-time system are often recurrent in nature. The sporadic task model is one of the most widely-studied notions of recurrent real-time task execution. In this model, each task is a sequential program that is invoked repeatedly; each such invocation is called a job and has an associated deadline by which it should complete execution.

It is generally required that jobs be scheduled so that they do not miss their deadlines. However, in a soft real-time system, deadline misses can sometimes be tolerated, if the amount by which a deadline is missed is within a specified per-task tardiness threshold. If $\delta$ is the tardiness threshold of some task, then its job with a deadline at time $d$ should be

\footnotetext{
*Work supported by NSF grants CCR 0309825 and CCR 0408996. The first author was also supported by an IBM Ph.D. fellowship.
}

guaranteed to complete execution by time $d+\delta$. Such a guarantee would ensure that, in the long run, each task receives a processor share commensurate with its utilization (see Sec. 2).

In work on real-time systems, multiprocessor platforms (SMPs) are of growing importance. This is due to both hardware trends such as the emergence of multicore technologies, and also to the prevalence of computationally-intensive applications for which single-processor designs are not sufficient. Examples of such applications include systems that track people and machines, many computer-vision systems, and signalprocessing applications such as synthetic aperture imaging. Timing constraints in several of these applications are predominantly soft. Given these observations, designing efficient scheduling algorithms for multiprocessor-based soft real-time systems and analyzing the traditional algorithms for soft realtime systems are goals of considerable value and interest.

Sporadic task systems can be scheduled on a multiprocessor using either a partitioning or a global-scheduling approach. Under partitioning, tasks are statically assigned to processors, and a uniprocessor scheduling algorithm is used on each processor to schedule its assigned tasks. In contrast, under global scheduling, a task may execute on any processor and may migrate across processors. Each approach can be differentiated further based on the scheduling algorithm that is used. For instance, the earliest-deadline-first (EDF) [6] or the rate-monotonic $(\mathrm{RM})^{*}[10]$ algorithm could be used as the per-processor scheduler under partitioning, or as the systemwide global scheduler.

Pfair scheduling [4], when deployed in a global setting, is currently the only known way of optimally scheduling sporadic task systems on a multiprocessor. ${ }^{\dagger}$ However, Pfair algorithms schedule tasks one quantum at a time, and as a result, jobs may be preempted and migrate across processors frequently. Such preemption and migration overheads can lower the amount of useful work that is actually accomplished. On the other hand, no known non-Pfair-based scheduling algorithm is optimal, and in the worst case, every such algorithm requires that the total utilization not exceed $(m+1) / 2$ (i.e.,

\footnotetext{
${ }^{*}$ Under RM scheduling, priorities for jobs are fixed offline and are inversely proportional to the periods of their tasks: the jobs of a task with a shorter period have higher priority than those of another task with a longer period.

†The term "optimal" means that on $m$ processors, such algorithms are capable of correctly scheduling (without any deadline misses) any task system with total utilization at most $m$.
} 
the underlying platform be underutilized by roughly $50 \%$ ), if every deadline is to be met $[9,11,3,2]$.

Prior work has shown that such restrictions on overall utilization can be eliminated for soft real-time systems. In [1], Anderson et al. presented a variant of partitioned-EDF that ensures bounded tardiness with no such restrictions, provided per-task utilizations are capped at $1 / 2$. In addition, in a recent paper [8], we derived tardiness bounds for both preemptive and non-preemptive global EDF. Tardiness bounds under EDF have also been presented by Valente and Lipari [13]. A precursor to all of the work mentioned here is a paper by Srinivasan and Anderson [12] in which tardiness bounds are presented for the earliest-pseudo-deadline first (EPDF) Pfair scheduling algorithm, which is sub-optimal but more efficient than optimal algorithms.

Contributions. The tardiness bounds derived by us previously for preemptive and non-preemptive EDF [8] are dependent on per-task utilizations and execution costs but independent of the total system utilization, $U_{\text {sum }}$. Furthermore, any task may incur maximum tardiness. This may not be acceptable to applications that are comprised of hard and soft realtime tasks with different tardiness tolerances. In this paper, we make an attempt to address this limitation.

Our contributions are twofold. First, we consider guaranteeing lower tardiness to some tasks at the expense of others. To this end, we propose a new scheduling policy, called EDF-hl, which is a variant of global EDF. We show that under EDF-hl on $m$ processors, up to $m$ tasks can be accorded preferential treatment and thereby guaranteed any tardiness, including zero, and that bounded tardiness can be guaranteed to the remaining tasks if their utilizations are capped. In the absence of tasks that require lower tardiness, EDF-hl reduces to EDF. Simulations involving randomly-generated task sets presented herein suggest that for many systems, the tardiness bounds that can be ensured for tasks that do not receive preferential treatment are acceptable.

Unlike in [8], the tardiness bound derived here is a function of $U_{\text {sum }}$, in addition to individual task parameters. Thus, as a second contribution, our bound offers the possibility of lowering tardiness for all tasks by lowering $U_{\text {sum }}$. To assess the tardiness-utilization trade-off for EDF (i.e., without special tasks), we again conducted experiments involving randomlygenerated task sets. We found that, with the improved analysis, considerable reductions in tardiness are possible even for reasonable reductions in total system utilization. For instance, in the simulation results for eight processors shown in Fig. 5(b) in Sec. 4, lowering $U_{\text {sum }}$ by around $10 \%$ results in a reduction in maximum tardiness by over $35 \%$, and lowering $U_{\text {sum }}$ by $25 \%$ lowers maximum tardiness by close to $50 \%$.

Organization. The rest of this paper is organized as follows. Our system model and algorithm EDF-hl are described in Sec. 2. Tardiness bounds are derived in Sec. 3. Results of the simulation-based evaluations mentioned above are presented in Sec. 4. Finally, Sec. 5 concludes.

\section{Definitions}

In this section, our task model is described and algorithm EDF-hl is presented.

Task model. A sporadic task system comprised of $n \geq 1$ sporadic tasks is to be scheduled on $m \geq 2$ processors. The $i^{\text {th }}$ task is denoted $T_{i}\left(e_{i}, p_{i}\right)$, where $p_{i}>0$ is the minimum inter-arrival separation for its successive jobs and $e_{i} \leq p_{i}$ is its per-job execution cost. $p_{i}$ is also referred to as the period of $T_{i}$. In the variant of the sporadic model considered here, $p_{i}$ is also the relative deadline of $T_{i}$, i.e., each job of $T_{i}$ must complete execution within $p_{i}$ time units of its invocation. The utilization of $T_{i}$ is given by $u_{i} \stackrel{\text { def }}{=} e_{i} / p_{i} \leq 1$. The total utilization of $\tau$ is defined as $U_{\text {sum }}(\tau) \stackrel{\text { def }}{=} \sum_{i=1}^{n} u_{i}$. It is required that $U_{\text {sum }}(\tau) \leq m$ hold. The maximum utilization (resp., execution cost) of any task in $\tau$ is denoted $u_{\max }(\tau)$ (resp., $e_{\max }(\tau)$ ). The minimum execution cost of any task is denoted $e_{\min }(\tau)$. (In this notation, the task system $\tau$ is omitted when unambiguous.)

The $k^{\text {th }}$ job of $T_{i}$, where $k \geq 1$, is denoted $T_{i, k}$, and its release time and absolute deadline (or simply deadline for short) are denoted $r_{i, k}$ and $d_{i, k}\left(=r_{i, k}+p_{i}\right)$, respectively. $r_{i, k}$ denotes the the time at or after which $T_{i, k}$ can be executed. $r_{i, k+1}-r_{i, k} \geq p_{i}$ holds for all $k \geq 1$. Each task is sequential, and hence no job of any task may execute in parallel. Furthermore, no two jobs of any task may execute in parallel.

A sporadic task system $\tau$ is said to be concrete if the release time of every job of each of its tasks is specified, and non-concrete, otherwise. Note that an infinite number of concrete task systems can be specified for every non-concrete task system. We omit specifying the type of the task system unless it is necessary. The results in this paper are for non-concrete task systems, and hence hold for every concrete task system.

The tardiness of a job $T_{i, j}$ in a schedule $\mathcal{S}$ is defined as $\operatorname{tardiness}\left(T_{i, j}, \mathcal{S}\right)=\max \left(0, t-d_{i, j}\right)$, where $t$ is the time at which $T_{i, j}$ completes executing in $\mathcal{S}$. If $\kappa$ is the maximum tardiness of any task system under $\mathcal{A}$, then $\mathcal{A}$ is said to ensure a tardiness bound of $\kappa$. We assume that missed deadlines do not delay future job releases. That is, even if a job of a task misses its deadline, the release time of the next job of that task remains unaltered. Since consecutive jobs of the same task cannot be scheduled in parallel, a missed deadline effectively reduces the interval over which the next job should be scheduled in order to meet its deadline.

The sporadic task model described above is augmented as follows for EDF-hl (described below). Each task in $\tau$ is classified as either a privileged task or an unprivileged task. The set of all privileged (resp., unprivileged) tasks is denoted $\tau_{H}$ (resp., $\tau_{L}$ ). ( $H$ and $L$ stand for high and low privilege.) $\left|\tau_{H}\right| \leq m$ holds. Each privileged task $T_{h}$ has a maximum tardiness parameter $\Delta_{h} \geq 0$, which denotes the maximum tardiness that any of its jobs can tolerate. $d_{h, j}+\Delta_{h}$ is referred to as the effective deadline of job $T_{h, j}$ and is denoted $\rho_{h, j}$.

To express the tardiness bound derived in this paper easily, we let $\epsilon_{i}, \epsilon_{i}^{H}$, and $\epsilon_{i}^{L}$ (resp., $\mu_{i}, \mu_{i}^{H}$, and $\mu_{i}^{L}$ ) denote the $i^{t h}$ 
execution cost (resp., task utilization) in a non-increasing ordering of those of tasks in $\tau, \tau_{H}$, and $\tau_{L}$, respectively. Also, $\gamma_{i}$ denotes the tardiness-utilization product, i.e., $\Delta_{i} \times u_{i}$, for the $i^{\text {th }}$ privileged task in a non-increasing ordering for tasks in $\tau_{H} . \Lambda$ is defined as follows.

$$
\Lambda= \begin{cases}U_{\text {sum }}(\tau)-1, & U_{\text {sum }}(\tau) \text { is integral } \\ \left\lfloor U_{\text {sum }}(\tau)\right\rfloor, & \text { otherwise }\end{cases}
$$

Algorithm EDF-hl. Our goal is to design an algorithm that can guarantee a tardiness of $\Delta_{h}$ to each privileged task $T_{h}$ while guaranteeing bounded tardiness to the remaining tasks. Let the slack of job $T_{h, j}$ of a privileged task $T_{h}$ at time $t$ be defined as $d_{h, j}+\Delta_{h}-t-\left(e_{h}-\delta_{h, j}\right)$, where $\delta_{h, j}$ denotes the amount of time that $T_{h, j}$ executed before $t$. Informally, the slack of job $T_{h, j}$ at $t$ is the amount of time the job can afford not to execute after $t$ until completion for its tardiness to be at most $\Delta_{h}$. A tardiness of at most $\Delta_{h}$ can be guaranteed to task $T_{h}$ if each job $T_{h, j}$ is scheduled based on its deadline until time $d_{h, j}+\Delta_{h}-e_{h}$, but is guaranteed continuous execution from $d_{h, j}+\Delta_{h}-e_{h}$ onward. (This is somewhat similar to the behavior of the earliest-deadline-until-zero-laxity algorithm described in [5].) Job $T_{h, j}$ is said to be urgent at time $t$, if $t \geq d_{h, j}+\Delta_{h}-e_{h}$ and $T_{h, j}$ has not completed execution by $t$. Note that $T_{h, j}$ is flagged as urgent from $d_{h, j}+\Delta_{h}-e_{h}$ until completion even if its slack is positive. This eliminates the overhead of updating the urgency for each privileged job at runtime and may result in fewer preemptions and migrations.

With the above definitions in place, Algorithm EDF-hl can be described as follows. At any time $t$, each of the urgent jobs, if any, of tasks in $\tau_{H}$ is assigned a unique processor. If not every processor is assigned to an urgent job, then the non-urgent jobs of $\tau_{H}$ and jobs of tasks in $\tau_{L}$ are scheduled on the remaining processors on an earliest-deadline-first basis, where ties, if any, are resolved arbitrarily. A job may be preempted at any time by a higher priority job and may later resume execution on a different processor.

Note that EDF-hl reduces to EDF if $\tau_{H}=\emptyset$. Since $\left|\tau_{H}\right| \leq m$ holds, EDF-hl clearly ensures the required tardiness for each privileged task. Hence, the question to be addressed is whether bounded tardiness can be guaranteed for the remaining tasks. The answer turns out to be yes if there is a cap on the utilizations of the remaining tasks. This cap depends on the number of privileged tasks and their utilizations. To see that such a cap is necessary, at least in some cases, consider a task system comprised of four tasks $T_{1}(3,4), \ldots, T_{3}(3,4)$, and $T_{4}(3 i, 4 i)$, where $i \geq 1$. Here, if tasks $T_{1}, \ldots, T_{3}$ require a tardiness of zero, then tardiness for $T_{4}$ can grow unboundedly.

Discussion. Though the tardiness bounds derived in [8] guarantee that tardiness for each task in the above example (with $i=1$ ) is at most 4.33 time units under EDF, no task is immune from incurring maximum tardiness. The bound for EDF-hl derived here would enable one of the four tasks to be guaranteed zero tardiness if the remaining tasks can tolerate a tardiness of 6 time units (which is only slightly higher than 4.33). However, if two tasks have a tardiness requirement of zero, then tardiness for the remaining tasks may be as high as 21.0 (which is still bounded). Lower tardiness can be guaranteed if the utilizations of the unprivileged tasks are lower. For instance, with two privileged tasks $T_{1}(3,4)$ and $T_{2}(3,4)$ and three unprivileged tasks $T_{3}(3,6), \ldots, T_{5}(3,6)$, the unprivileged tasks can be guaranteed a bound of 12.0.

\section{Tardiness under EDF-hl}

In this section, we determine a tardiness bound for $\tau_{L}$. The approach for doing this is the same as that used in [8]. This involves comparing the allocations to a concrete task system $\tau$ in a processor sharing (PS) schedule for $\tau$ and an actual EDF-hl schedule of interest for $\tau$, and quantifying the difference between the two. In a PS schedule, each job of $T_{i}$ is allocated a fraction $u_{i}$ of a processor at each instant (or equivalently, a fraction $u_{i}$ of each instant) in the interval between its release time and its deadline. Because $U_{\text {sum }} \leq m$ holds, the total demand at any instant will not exceed $m$ in a PS schedule, and hence no deadlines will be missed; in fact, every job will complete executing exactly at its deadline. We begin by setting the required machinery in place.

\subsection{Definitions and Notation}

A time interval $\left[t_{1}, t_{2}\right)$, where $t_{2} \geq t_{1}$, consists of all times $t$, where $t_{1} \leq t<t_{2}$, and is of length $t_{2}-t_{1}$. The system start time is assumed to be zero. For any time $t>0, t^{-}$denotes the time $t-\epsilon$ in the limit $\epsilon \rightarrow 0+$.

Definition 1 (active tasks and active jobs): $\mathrm{A}$ task $T_{i}$ is said to be active at time $t$, if there exists a job $T_{i, j}$ (called $T_{i}$ 's active job at $t$ ) such that $r_{i, j} \leq t<d_{i, j}$. By our task model, every task can have at most one active job at any time.

Definition 2 (pending jobs): $T_{i, j}$ is said to be pending at $t$ in a schedule $\mathcal{S}$ if $r_{i, j} \leq t$ and $T_{i, j}$ has not completed execution by $t$ in $\mathcal{S}$. Note that a job with a deadline at or before $t$ is not considered to be active at $t$ even if it is pending at $t$.

Definition 3 (ready jobs): A pending job $T_{i, j}$ is said to be ready at $t$ in a schedule $\mathcal{S}$ if all prior jobs of $T_{i}$ have completed execution by $t$ in $\mathcal{S}$.

We now quantify the total allocation to $\tau$ in an interval $\left[t_{1}, t_{2}\right)$ in a PS schedule for $\tau, \mathrm{PS}_{\tau}$. Let $\mathrm{A}\left(\mathcal{S}, T_{i}, t_{1}, t_{2}\right)$ denote the total time allocated to $T_{i}$ in an arbitrary schedule $\mathcal{S}$ for $\tau$ in $\left[t_{1}, t_{2}\right)$. Then, since in $\mathrm{PS}_{\tau}, T_{i}$ is allocated a fraction $u_{i}$ of each instant at which it is active in $\left[t_{1}, t_{2}\right)$, we have

$$
\mathrm{A}\left(\mathrm{PS}_{\tau}, T_{i}, t_{1}, t_{2}\right) \leq\left(t_{2}-t_{1}\right) u_{i} .
$$

The total allocation to $\tau$ in the same interval in $\mathrm{PS}_{\tau}$ is

$$
\mathrm{A}\left(\mathrm{PS}_{\tau}, \tau, t_{1}, t_{2}\right) \leq \sum_{T_{i} \in \tau}\left(t_{2}-t_{1}\right) u_{i}=U_{\text {sum }}(\tau) \cdot\left(t_{2}-t_{1}\right)
$$

We are now ready to define lag and LAG, which play a pivotal role in this paper. The lag of task $T_{i}$ at time $t$ in schedule $\mathcal{S}$, denoted $\operatorname{lag}\left(T_{i}, t, \mathcal{S}\right)$, is given by

$$
\operatorname{lag}\left(T_{i}, t, \mathcal{S}\right)=\mathrm{A}\left(\mathrm{PS}_{\tau}, T_{i}, 0, t\right)-\mathrm{A}\left(\mathcal{S}, T_{i}, 0, t\right) .
$$


In $\mathcal{S}$, less work than in $\mathrm{PS}_{\tau}$ has been completed by time $t$ on the jobs of $T_{i}$ if $\operatorname{lag}\left(T_{i}, t, \mathcal{S}\right)$ is positive (i.e., $T_{i}$ is underallocated in $\mathcal{S}$ ), and more work, if $\operatorname{lag}\left(T_{i}, t, \mathcal{S}\right)$ is negative (i.e., $T_{i}$ is over-allocated in $\mathcal{S}$ ). The total lag of a task system $\tau$ at $t$, denoted $\operatorname{LAG}(\tau, t, \mathcal{S})$, is given by

$$
\begin{aligned}
\operatorname{LAG}(\tau, t, \mathcal{S}) & =\sum_{T_{i} \in \tau} \operatorname{lag}\left(T_{i}, t, \mathcal{S}\right) \\
& =\mathrm{A}\left(\mathrm{PS}_{\tau}, \tau, 0, t\right)-\mathrm{A}(\mathcal{S}, \tau, 0, t) .
\end{aligned}
$$

Note that $\operatorname{LAG}(\tau, 0, \mathcal{S})$ and $\operatorname{lag}\left(T_{i}, 0, \mathcal{S}\right)$ are both zero, and that by (4) and (5), we have the following for $t_{2}>t_{1}$.

$$
\begin{gathered}
\operatorname{lag}\left(T_{i}, t_{2}, \mathcal{S}\right)=\operatorname{lag}\left(T_{i}, t_{1}, \mathcal{S}\right)+ \\
\mathrm{A}\left(\mathrm{PS}_{\tau}, T_{i}, t_{1}, t_{2}\right)-\mathrm{A}\left(\mathcal{S}, T_{i}, t_{1}, t_{2}\right) \\
\operatorname{LAG}\left(\tau, t_{2}, \mathcal{S}\right)=\operatorname{LAG}\left(\tau, t_{1}, \mathcal{S}\right)+ \\
\mathrm{A}\left(\mathrm{PS}_{\tau}, \tau, t_{1}, t_{2}\right)-\mathrm{A}\left(\mathcal{S}, \tau, t_{1}, t_{2}\right)
\end{gathered}
$$

Lag for jobs. The notion of lag defined above for tasks and task sets can be applied to jobs and job sets in an obvious manner. Let $\tau$ denote a concrete task system, and $\Psi$ a subset of jobs in $\tau$. Let $\mathrm{A}\left(\mathrm{PS}_{\tau}, T_{i, j}, t_{1}, t_{2}\right)$ and $\mathrm{A}\left(\mathcal{S}, T_{i, j}, t_{1}, t_{2}\right)$ denote the allocations to $T_{i, j}$ in $\left[t_{1}, t_{2}\right)$ in $\mathrm{PS}_{\tau}$ and $\mathcal{S}$, respectively. Then, $\operatorname{lag}\left(T_{i, j}, t, \mathcal{S}\right)=$ $\mathrm{A}\left(\mathrm{PS}_{\tau}, T_{i, j}, r_{i, j}, t\right)-\mathrm{A}\left(\mathcal{S}, T_{i, j}, r_{i, j}, t\right)$, and $\operatorname{LAG}(\Psi, t, \mathcal{S})=$ $\sum_{T_{i, j} \in \Psi} \operatorname{lag}\left(T_{i, j}, t, \mathcal{S}\right)$. The total allocation in $[0, t)$, where $t>0$, to a job that is neither pending at $t^{-}$in $\mathcal{S}$ nor is active at $t^{-}$is the same in both $\mathcal{S}$ and $\mathrm{PS}_{\tau}$, and hence, its lag at $t$ is zero. Therefore, for $t>0$, we have

$$
\operatorname{LAG}(\Psi, t, \mathcal{S})=\sum_{\begin{array}{l}
\left\{T_{i, j} \text { is in } \Psi,\right. \text { and is pending } \\
\text { or active at } \left.t^{-}\right\}
\end{array}} \operatorname{lag}\left(T_{i, j}, t, \mathcal{S}\right) .
$$

The above expression can be rewritten using task lags as follows (since no job can be scheduled before its release time and jobs of $T_{i}$ that are not in $\Psi$ may be pending at $t$ ).

$$
\operatorname{LAG}(\Psi, t, \mathcal{S}) \leq \sum_{\substack{\left\{T_{i} \in \tau: T_{i, j} \text { is in } \Psi, \text { and is } \\ \text { pending or active at } t^{-}\right\}}} \operatorname{lag}\left(T_{i}, t, \mathcal{S}\right)
$$

Similarly, the total utilization of $\Psi$ at time $t$ is given by the sum of the utilizations of tasks with an active job at $t$ in $\Psi$ :

$$
U_{\text {sum }}(\Psi, t)=\sum_{\left\{T_{i} \in \tau: T_{i, j} \text { is in } \Psi\right. \text { and is ac- }} u_{i} .
$$

Definition 4 (busy interval): A time interval $\left[t_{1}, t_{2}\right)$, where $t_{2}>t_{1}$, is said to be busy for $\tau$ if all $m$ processors are executing jobs of tasks in $\tau$ throughout the interval, i.e., no processor is ever idle in the interval or executes a job of a task not in $\tau$. An interval $\left[t_{1}, t_{2}\right)$ that is not busy for $\tau$ is said to be nonbusy for $\tau$, and is maximally non-busy if every time instant in $\left[t_{1}, t_{2}\right)$ is non-busy, and either $t_{1}=0$ or $t_{1}^{-}$is busy.

If at least $U_{\text {sum }}(\tau)$ tasks are executing at every instant in $\left[t_{1}, t_{2}\right)$ in a schedule $\mathcal{S}$ for $\tau$, then the tasks in $\tau$ receive a total allocation of $U_{\text {sum }}(\tau) \cdot\left(t_{2}-t_{1}\right)$ time in $\mathcal{S}$ in that interval. By (3), the total allocation to $\tau$ in $\left[t_{1}, t_{2}\right)$ cannot exceed $U_{\text {sum }}(\tau)$. $\left(t_{2}-t_{1}\right)$ in $\mathrm{PS}_{\tau}$. Therefore, by (6), the LAG of $\tau$ at $t_{2}$ cannot exceed that at $t_{1}$, and we have the following lemma.
Lemma 1 If $\operatorname{LAG}(\tau, t+\delta, \mathcal{S})>\operatorname{LAG}(\tau, t, \mathcal{S})$, where $\delta>0$ and $\mathcal{S}$ is a schedule for $\tau$, then $[t, t+\delta)$ is a non-busy interval for $\tau$. Furthermore, there exists at least one instant in $[t, t+\delta)$ at which fewer than $U_{\text {sum }}(\tau)$ tasks are executing.

The busy interval in Def. 4 is defined with respect to $\tau$. With respect to $\Psi,\left[t_{1}, t_{2}\right)$ is said to be busy only if every processor is executing some job of $\Psi$ throughout $\left[t_{1}, t_{2}\right)$. The job-set counterpart of Lemma 1 is as follows.

Lemma 2 If $\operatorname{LAG}(\Psi, t+\delta, \mathcal{S})>\operatorname{LAG}(\Psi, t, \mathcal{S})$, where $\delta>0$ and $\mathcal{S}$ is a schedule for $\tau$, then $[t, t+\delta)$ is a non-busy interval for $\Psi$. Furthermore, there exists at least one instant $t^{\prime}$ in $[t, t+$ $\delta)$ at which fewer than $U_{\text {sum }}\left(\Psi, t^{\prime}\right)$ tasks are executing.

\subsection{Deriving a Tardiness Bound}

Given an arbitrary non-concrete task system $\tau^{N}$, we are interested in determining the highest tardiness of any job of any task in $\tau_{L}^{N}$ in any concrete instantiation of $\tau^{N}$. Let $\tau$ (resp., $\tau_{H}$ and $\tau_{L}$ ) be a concrete instantiation of $\tau^{N}$ (resp., $\tau_{H}^{N}$ and $\left.\tau_{L}^{N}\right), T_{\ell, j}$ a job in $\tau_{L}, t_{d}=d_{\ell, j}$, and $\mathcal{S}$ an EDF-hl schedule for $\tau$ with the following property.

(P) The tardiness of every job of every task $T_{k}$ in $\tau_{L}$ with deadline less than $t_{d}$ is at most $x+e_{k}$, where $x \geq 0$.

Then, determining the smallest $x$, independent of the parameters of $T_{\ell}$, such that the tardiness of $T_{\ell, j}$ remains at most $x+e_{\ell}$ would by induction imply a tardiness of at most $x+e_{k}$ for all jobs of tasks in $\tau_{L}$. Because $\tau$ is arbitrary, the tardiness bound will hold for every concrete instantiation of $\tau^{N}$.

Our proof obligation is easily met if $T_{\ell, j}$ completes by its deadline, $t_{d}$, so assume otherwise. The completion time of $T_{\ell, j}$ depends on the amount of work that can compete with $T_{\ell, j}$ after $t_{d}$. We follow the steps below to determine $x$.

(S1) Compute an upper bound (UB) on the amount of work (including that due to $T_{\ell, j}$ ) that can compete with $T_{\ell, j}$ after $t_{d}$.

(S2) Determine a lower bound (LB) on the amount of such work required for the tardiness of $T_{\ell, j}$ to exceed $x+e_{\ell}$.

(S3) Determine the smallest $x$ such that the tardiness of $T_{\ell, j}$ is at most $x+e_{\ell}$ using UB and LB.

Let $\Psi$ denote the set of all jobs with deadlines at most $t_{d}$ of all tasks in $\tau$. Under EDF-hl, no job of a task in $\tau_{L}$ with a deadline after $t_{d}$ can compete with $T_{\ell, j}$. Therefore, competing work for $T_{\ell, j}$ is given by (i) the amount of work pending at $t_{d}$ for jobs in $\Psi$, i.e., $\operatorname{LAG}\left(\Psi, t_{d}, \mathcal{S}\right)$, plus (ii) the amount of work demanded by jobs of tasks in $\tau_{H}$ that are not in $\Psi$ but can compete with jobs in $\Psi$ in $\left[t_{d}, t_{d}+x+e_{\ell}\right)$. We now determine an upper bound on these two components (step (S1) above).

(In the analysis that follows, we assume that $\Delta_{h} \ll x$ holds for all $T_{h}$ in $\tau_{H}$. The analysis has to be extended slightly, otherwise. We have refrained from presenting a more general analysis in the interest of clarity.) 


\subsubsection{Upper Bound on $\operatorname{LAG}\left(\Psi, t_{d}, \mathcal{S}\right)$}

Let the carry-in job of a task $T_{h}$ in $\tau_{H}$ be defined as that job, if any, of $T_{h}$, with a release time before $t_{d}$ and an absolute deadline afterward. Clearly, at most one such job exists for each $T_{h}$. Similarly, let the job, if any, of $T_{h}$, with a release time before $t_{d}+x+e_{\ell}$ and an effective deadline afterward be defined as its carry-out job. This is illustrated in Fig. 3. The carry-in job of $T_{h}$ is its only job with an absolute deadline after $t_{d}$ that may preempt (i.e., compete with) jobs in $\Psi$ before $t_{d}$ (i.e., become urgent before $t_{d}$ ). Let $\Psi_{H}$ be the set of all carry-in jobs of tasks in $\tau_{H}$. (For easy reference, descriptions for these task and job sets are repeated in Fig. 1.)

\section{By Lemma 2,} the LAG of $\Psi$ can increase only across a non-busy interval for $\Psi$. Recall that in a non-busy interval for $\Psi$ fewer than $m$

\begin{tabular}{|c|l|}
\hline $\begin{array}{c}\text { Task / } \\
\text { Job Set }\end{array}$ & \multicolumn{1}{c|}{ Description } \\
\hline \hline$\tau_{H}$ & Set of all privileged tasks in $\tau$ \\
\hline$\tau_{L}$ & Set of all unprivileged tasks in $\tau$ \\
\hline$\Psi$ & $\begin{array}{l}\text { Set of all jobs of all tasks in } \tau \\
\text { with deadline at most } t_{d}\end{array}$ \\
\hline$\Psi_{H}$ & Set of carry-in jobs of tasks in $\tau_{H}$ \\
\hline
\end{tabular}

Figure 1. Task and job sets heavily referred to.

jobs from $\Psi$ execute. In the case of an EDF-hl schedule, such a non-busy interval for $\Psi$ can be classified into two types depending on whether a job from $\Psi_{H}$ is executing in the interval while a ready job from $\Psi$ is waiting. At the risk of slightly abusing terms, we will refer to the two types as blocking and non-blocking non-busy intervals. A blocking, non-busy interval is one in which a job from $\Psi_{H}$ is executing while a ready job from $\Psi$ is waiting, whereas a non-blocking, non-busy interval is one in which fewer than $m$ jobs from $\Psi$ are executing, but there does not exist a ready job in $\Psi$ that is waiting. Note that it is immaterial whether a job from $\Psi_{H}$ is executing in a non-blocking, non-busy interval.

Before determining an upper bound on LAG, we state some needed properties. In [8], we showed that if a task does not execute continuously within a non-busy interval in an EDF schedule, then its lag at the end of the interval is at most zero. This property can be extended to a non-blocking, non-busy interval of an EDF-hl schedule, as follows.

Lemma 3 (from $[\mathbf{8}]$ ) Let $\left[t, t^{\prime}\right.$ ) be a maximally non-blocking, non-busy interval in $\left[0, t_{d}\right)$ in $\mathcal{S}$ and let $T_{k}$ be a task in $\tau$ with a job in $\Psi$ that is active or pending at $t^{\prime-}$. If $T_{k}$ does not execute continuously in $\left[t, t^{\prime}\right)$, then $\operatorname{lag}\left(T_{k}, t^{\prime}, \mathcal{S}\right) \leq 0$.

The two lemmas that follow are proved in [7]. The first lemma bounds the lag of a task in $\tau_{L}$ at any arbitrary time at or before $t_{d}$. The second concerns the lags of tasks in $\tau_{H}$.

Lemma 4 Let $v$ be an arbitrary time instant at or before $t_{d}$. Let $T_{k}$ be a task in $\tau_{L}$ and $T_{k, q}$ its earliest pending job at $v$, and let $\delta_{k, q}<e_{k}$ be the amount of time that $T_{k, q}$ executed for before $v$. Then, $\operatorname{lag}\left(T_{k}, v, \mathcal{S}\right) \leq\left(v-d_{k, q}\right) \cdot u_{k}+e_{k}-\delta_{k, q}$. Furthermore, $v-d_{k, q} \leq x+\delta_{k, q}$. Hence, $\operatorname{lag}\left(T_{k}, v, \mathcal{S}\right) \leq$ $x \cdot u_{k}+e_{k}$.
Lemma 5 Let $T_{k}$ be a task in $\tau_{H}$ and $T_{k, q}$ its earliest pending job at any arbitrary time $v$. Then, $\operatorname{lag}\left(T_{k}, v, \mathcal{S}\right) \leq \min \left(d_{k, q}+\right.$ $\left.\Delta_{k}-v, e_{k}\right)+\left(v-d_{k, q}\right) \cdot u_{k} \leq e_{k}+\Delta_{k} \cdot u_{k}$.

We now turn to determining an upper bound on the LAG of $\Psi$ at $t_{d}$. By Lemma 2, the LAG of $\Psi$ can increase only across a non-busy interval for $\Psi$. Hence,

\begin{tabular}{|c|l|}
\hline Symbol & \multicolumn{1}{|c|}{ Expression } \\
\hline \hline$E_{L}$ & $\sum_{k=1}^{\Lambda} \epsilon_{k}$ \\
\hline$E_{H}$ & $\sum_{T_{h} \in \tau_{H}} e_{h}\left(1-u_{h}\right)$ \\
\hline$U_{L}$ & $\sum_{k=1}^{\min \left(\Lambda-1,\left|\tau_{L}\right|\right)} \mu_{k}^{L}$ \\
\hline$U_{H}$ & $\sum_{h=1}^{\max \left(0, \Lambda-1-\left|\tau_{L}\right|\right)_{h}} \gamma_{h}$ \\
\hline$U_{H}^{\prime}$ & $\sum_{T_{h} \in \tau_{H}} u_{h}$ \\
\hline$E_{H}^{\prime}$ & $\begin{array}{l}\sum_{T_{h} \in \tau_{H}}\left(\left(e_{h}\left(1-u_{h}\right)+\right.\right. \\
\left.u_{h}\left(e_{\ell}-\Delta_{h}\right)+\min \left(e_{h} \cdot u_{h}, \Delta_{h}\right)\right) \\
\\
\left.+\max \left(u_{h}\left(e_{h}-e_{\ell}\right), 0\right)\right)\end{array}$ \\
\hline
\end{tabular}

Figure 2. Frequently referred-to expressions.

an upper bound on LAG at the end of the latest non-busy interval before $t_{d}$ across which LAG increases will serve as an upper bound for that at $t_{d}$. As discussed earlier, a non-busy interval in an EDF-hl schedule can be either blocking or non-blocking. We will consider these two cases separately. Expressions that occur frequently in the analysis are provided in Fig. 2. The lemma that follows shows how to bound LAG at the end of a non-blocking, non-busy interval.

Lemma 6 Let $\left[t, t^{\prime}\right)$ be a maximally non-blocking, non-busy interval in $\left[0, t_{d}\right)$ in $\mathcal{S}$ and let $\mathrm{LAG}\left(\Psi, t^{\prime}, \mathcal{S}\right)>\operatorname{LAG}(\Psi, t, \mathcal{S})$. Then, $\operatorname{LAG}\left(\Psi, t^{\prime}, \mathcal{S}\right) \leq x \cdot U_{L}+U_{H}+E_{L}$.

Proof: By (7), the LAG of $\Psi$ at $t^{\prime}$ is given by the sum of the lags at $t^{\prime}$ of all tasks in $\tau$ with at least one job in $\Psi$ that is active or pending at $t^{\prime-}$. By Lemma 3, the lag of such a task that does not execute continuously in $\left[t, t^{\prime}\right)$ is at most zero. Hence, to determine an upper bound on LAG at $t^{\prime}$, it is sufficient to determine an upper bound on the lags of such tasks that are executing continuously in $\left[t, t^{\prime}\right)$. Let $\Lambda^{\prime}$ denote the number of such tasks. Then, by Lemma 2 ,

$$
\Lambda^{\prime}<\max _{t \leq \hat{t}<t^{\prime}}\left\{U_{\text {sum }}(\Psi, \hat{t})\right\} \leq U_{\text {sum }}(\tau) .
$$

Let $\alpha_{H}$ (resp., $\alpha_{L}$ ) denote the subset of all tasks in $\tau_{H}$ (resp., $\tau_{L}$ ) that are executing continuously in $\left[t, t^{\prime}\right)$ and have a job in $\Psi$ that is active or pending at $t^{\prime-}$. Then,

$$
\left|\alpha_{H}\right|+\left|\alpha_{L}\right|=\Lambda^{\prime},
$$

and by the above discussion on bounding LAG,

$$
\begin{aligned}
\operatorname{LAG}\left(\Psi, t^{\prime}, \mathcal{S}\right) & \leq \sum_{T_{h} \in \alpha_{H}} \operatorname{lag}\left(T_{h}, t^{\prime}, \mathcal{S}\right)+\sum_{T_{k} \in \alpha_{L}} \operatorname{lag}\left(T_{k}, t^{\prime}, \mathcal{S}\right) \\
\leq & \sum_{T_{h} \in \alpha_{H}}\left(\Delta_{h} \cdot u_{h}+e_{h}\right)+\sum_{T_{k} \in \alpha_{L}}\left(x \cdot u_{k}+e_{k}\right) \\
= & \sum_{T_{k} \in \alpha_{L} \cup \alpha_{H}} e_{k}+\sum_{T_{k} \in \alpha_{L}} x \cdot u_{k}+\sum_{T_{h} \in \alpha_{H}} \Delta_{h} \cdot u_{h}
\end{aligned}
$$




$$
\begin{aligned}
& \leq \sum_{k=1}^{\Lambda^{\prime}} \epsilon_{k}+\sum_{T_{k} \in \alpha_{L}} x \cdot u_{k}+\sum_{T_{h} \in \alpha_{H}} \Delta_{h} \cdot u_{h} \quad\{\mathrm{By}(10)\} \\
& \leq \sum_{k=1}^{\Lambda^{\prime}} \epsilon_{k}+\sum_{k=1}^{\min \left(\Lambda^{\prime},\left|\tau_{L}\right|\right)} x \cdot \mu_{k}^{L}+\sum_{h=1}^{\max \left(0, \Lambda^{\prime}-\left|\tau_{L}\right|\right)} \gamma_{h}
\end{aligned}
$$

\{By (10) and assuming $\Delta_{h} \ll x$ so that

$\Delta_{h} \cdot u_{h}<x \cdot u_{k}$ for all $\left.T_{h} \in \tau_{H}, T_{k} \in \tau_{L}\right\}$

Finally, as in [8], it can be shown that for LAG to increase across $\left[t, t^{\prime}\right)$, at least one job of $\Psi$ with a deadline at or after $t^{\prime}$ should have completed execution before $t$ and that at least one job executing at $t$ should have a deadline at or after $t^{\prime}$. Hence, the lag for its task $T_{k}$ at $t^{\prime}$ is at most $e_{k}$. By this argument, the upper bound on LAG derived above reduces to

$$
\begin{aligned}
& \operatorname{LAG}\left(\Psi, t^{\prime}, \mathcal{S}\right) \\
& \leq \sum_{k=1}^{\Lambda^{\prime}} \epsilon_{k}+\sum_{k=1}^{\min \left(\Lambda^{\prime}-1,\left|\tau_{L}\right|\right)} x \cdot \mu_{k}^{L}+\sum_{h=1}^{\max \left(0, \Lambda^{\prime}-1-\left|\tau_{L}\right|\right)} \gamma_{h} .
\end{aligned}
$$

The lemma follows because, by (1) and (9), $\Lambda^{\prime} \leq \Lambda$.

The next lemma shows how to bound LAG at the end of a blocking, non-busy interval.

Lemma 7 Let $\left[t, t^{\prime}\right)$ be a blocking, non-busy interval in $\left[0, t_{d}\right)$ in $\mathcal{S}$ such that every instant in $\left[t, t^{\prime}\right)$ is a blocking instant and any job of $\Psi_{H}$ that executes in $\left[t, t^{\prime}\right)$ executes continuously in $\left[t, t^{\prime}\right)$. Then, $\operatorname{LAG}\left(\Psi, t^{\prime}, \mathcal{S}\right) \leq \operatorname{LAG}(\Psi, t, \mathcal{S})+\sum_{T_{h} \in \alpha_{H}}\left(t^{\prime}-\right.$ $t) \cdot\left(1-u_{h}\right)$, where $\alpha_{H}$ is the subset of all tasks in $\tau_{H}$ whose jobs in $\Psi_{H}$ execute continuously in $\left[t, t^{\prime}\right)$.

Proof: Let $T_{h}$ be a task in $\alpha_{H}$, where $\alpha_{H}$ is as defined in the statement of the lemma. Then, because the job of $T_{h}$ that is executing in $\left[t, t^{\prime}\right)$ is in $\Psi_{H}, T_{h}$ does not have a job in $\Psi$ that is either active or pending anywhere in $\left[t, t^{\prime}\right)$. Thus, by (8),

$$
\left(\forall \hat{t}: t \leq \hat{t}<t^{\prime}:: U_{\text {sum }}(\Psi, \hat{t}) \leq U_{\text {sum }}(\tau)-\sum_{T_{h} \in \alpha_{H}} u_{h}\right),
$$

and since the cumulative allocation at each instant in $\left[t, t^{\prime}\right)$ in $\mathrm{PS}_{\tau}$ to jobs in $\Psi$ is at most $U_{\text {sum }}(\tau)-\sum_{T_{h} \in \alpha_{H}} u_{h}$, the following holds.

$$
\mathrm{A}\left(\mathrm{PS}_{\tau}, \Psi, t, t^{\prime}\right) \leq\left(t^{\prime}-t\right) \cdot\left(U_{\text {sum }}(\tau)-\sum_{T_{h} \in \alpha_{H}} u_{h}\right)
$$

Because $\left[t, t^{\prime}\right)$ is continuously blocking, at every instant in $\left[t, t^{\prime}\right)$, there exists at least one job in $\Psi$ that is ready, but does not execute. This in turn implies that no processor is idle in the interval. Hence, we have the following.

$$
\mathrm{A}\left(\mathcal{S}, \Psi, t, t^{\prime}\right)=\left(t^{\prime}-t\right) \cdot\left(m-\left|\alpha_{H}\right|\right)
$$

By (12) and (13), and (6) (with $t_{1}=t$ and $t_{2}=t^{\prime}$ ), we have

$$
\begin{aligned}
& \operatorname{LAG}\left(\Psi, t^{\prime}, \mathcal{S}\right) \\
& \leq \operatorname{LAG}(\Psi, t, \mathcal{S})+ \\
& \quad\left(t^{\prime}-t\right)\left(\left(U_{\text {sum }}(\tau)-\sum_{T_{h} \in \alpha_{H}} u_{h}\right)-\left(m-\left|\alpha_{H}\right|\right)\right)
\end{aligned}
$$

$$
\begin{aligned}
= & \operatorname{LAG}(\Psi, t, \mathcal{S})+ \\
& \left.\left(t^{\prime}-t\right)\left(\left(U_{\text {sum }}(\tau)-m\right)+\left|\alpha_{H}\right|-\sum_{T_{h} \in \alpha_{H}} u_{h}\right)\right) .
\end{aligned}
$$

Because $U_{\text {sum }}(\tau) \leq m$, the above implies $\operatorname{LAG}\left(\Psi, t^{\prime}, \mathcal{S}\right) \leq$ $\operatorname{LAG}(\Psi, t, \mathcal{S})+\left(t^{\prime}-t\right) \cdot \sum_{T_{h} \in \alpha_{H}}\left(1-u_{h}\right)$.

An upper bound on the LAG of $\Psi$ at $t_{d}$ can be determined by combining Lemmas 6 and 7 as follows. (This lemma is proved in [7].)

Lemma 8 Let $\delta_{h} \leq e_{h}$ denote the amount of time that the carry-in job (i.e., job in $\Psi_{H}$ ), if any, of task $T_{h}$ in $\tau_{H}$ executes for before $t_{d}$. Then, $\operatorname{LAG}\left(\Psi, t_{d}, \mathcal{S}\right) \leq x \cdot U_{L}+U_{H}+E_{L}+$ $\sum_{T_{h} \in \tau_{H}} \delta_{h} \cdot\left(1-u_{h}\right)$.

To complete step (S1), we need to determine an upper bound on the work due to jobs of tasks in $\tau_{H}$ that can compete with jobs in $\Psi$ in $\left[t_{d}, t_{d}+x+e_{\ell}\right)$. We do this next.

\subsubsection{Competing Demand due to Jobs of $\tau_{H}$ not in $\Psi$}

Let $\mathrm{D}\left(T_{h}\right)$ denote the amount of work due to the jobs of a task $T_{h}$ in $\tau_{H}$ that are not in $\Psi$ and that can compete with jobs of other tasks in $\Psi$ in $\left[t_{d}, t_{d}+x+e_{\ell}\right)$. Then, $\mathrm{D}\left(T_{h}\right)$ is composed of three parts: (i) Work that needs to be done on a carry-in job, if any, (ii) mandatory work that needs to be done on a carry-out job, if any, and (iii) work to be done on all jobs that lie between the carry-in and carry-out jobs, which is $e_{h}$ times the number of such jobs. This is illustrated in Fig. 3. (Note that because the effective deadlines of any two consecutive jobs of $T_{h}$ are separated by at least $p_{h}$ time units, the latter of any two such jobs does not become urgent until after the effective deadline of the former job has elapsed. Hence, no job released after the carry out job can compete with a job in $\Psi$ in $\left[t_{d}, t_{d}+x+e_{\ell}\right)$.)

We now derive a bound on $\mathrm{D}\left(T_{h}\right)$.

Lemma 9 Let $T_{h}$ be any task in $\tau_{H}$. Then, $\mathrm{D}\left(T_{h}\right) \leq e_{h}-$ $\delta_{h}+u_{h} \cdot\left(x+e_{\ell}-\Delta_{h}\right)+\min \left(0, \Delta_{h}-\left(e_{h}-\delta_{h}\right) u_{h}\right)+$ $\max \left(0, u_{h}\left(e_{h}-e_{\ell}\right)\right)$, where $\delta_{h} \leq e_{h}$ is the amount of time that the carry-in job, if any, of $T_{h}$ executes before $t_{d}$.

Proof: If no job of $T_{h}$ has its effective deadline in $\left[t_{d}, t_{d}+x+\right.$ $\left.e_{\ell}\right)$, then at most one job of $T_{h}$ executes in the interval, and the maximum amount of time it executes for cannot exceed $e_{h}-\delta_{h}$. Therefore, $\mathrm{D}\left(T_{h}\right) \leq e_{h}-\delta_{h}$ holds. Assuming $\Delta_{h} \ll$ $x$, it can be shown that $u_{h} \cdot\left(x+e_{\ell}-\Delta_{h}\right)+\min \left(0, \Delta_{h}-\left(e_{h}-\right.\right.$ $\left.\left.\delta_{h}\right) u_{h}\right)+\max \left(0, u_{h}\left(e_{h}-e_{\ell}\right)\right) \geq 0$ holds. Hence, $\mathrm{D}\left(T_{h}\right) \leq$ $e_{h}-\delta_{h}+u_{h} \cdot\left(x+e_{\ell}-\Delta_{h}\right)+\min \left(0, \Delta_{h}-\left(e_{h}-\delta_{h}\right) u_{h}\right)+$ $\max \left(0, u_{h}\left(e_{h}-e_{\ell}\right)\right)$, which proves the lemma.

Hence, for the rest of the proof assume that at least one job of $T_{h}$ has its effective deadline in $\left[t_{d}, t_{d}+x+e_{\ell}\right)$. Let $T_{h, c_{i}}$ and $T_{h, c_{o}}$ denote the carry-in and carry-out jobs, if any, of $T_{h}$.

Let $\xi_{h}=\rho_{h, c_{i}}-t_{d}$ and let $\phi_{h}$ denote the offset from $t_{d}+$ $x+e_{\ell}$ of the last effective deadline in $\left[t_{d}, t_{d}+x+e_{\ell}\right)$ of a job of $T_{h}$. Refer to Fig. 3. We now determine the three components of $\mathrm{D}\left(T_{h}\right)$ mentioned above. 


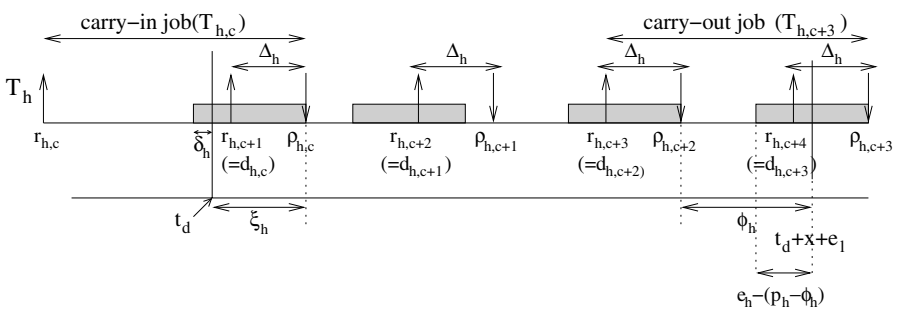

Figure 3. Competing demand due to task $T_{h}$ in $\tau_{H}$ in the interval $\left[t_{d}, t_{d}+x+e_{\ell}\right)$. Competing demand due to the carry-in job $T_{h, c_{i}}$ ( $T_{h, c}$ here) is at most $\min \left(e_{h}-\delta_{h}, \xi_{h}\right)$ and that due to the carry-out job $T_{h, c_{o}}\left(T_{h, c+3}\right.$ here $)$ is at most $\max \left(0, e_{h}-\left(p_{h}-\phi_{h}\right)\right)$.

Work due to $\boldsymbol{T}_{\boldsymbol{h}, \boldsymbol{c}_{\boldsymbol{i}}}$. Since $T_{h, c_{i}}$ completes executing by $\rho_{h, c_{i}}$, the amount of time that $T_{h, c_{i}}$ can execute for after $t_{d}$ is at most $\rho_{h, c_{i}}-t_{d}=\xi_{h}$ time units. Because $T_{h, c_{i}}$ executes for $\delta_{h}$ time units before $t_{d}$, it cannot execute for more than $e_{h}-\delta_{h}$ time units after $t_{d}$. Thus, the amount of work to be done on $T_{h, c_{i}}$ after $t_{d}$ is at most $\min \left(e_{h}-\delta_{h}, \xi_{h}\right)$.

Work due to $\boldsymbol{T}_{\boldsymbol{h}, c_{o}}$. The effective deadline of $T_{h, c_{o}}$ is separated from the previous effective deadline of $T_{h}$ by at least $p_{h}$ time units. Since the last effective deadline within $\left[t_{d}, t_{d}+x+e_{\ell}\right)$ is $\phi_{h}$ time units before $t_{d}+x+e_{\ell}, \rho_{h, c_{o}}$ is at least $p_{h}-\phi_{h}$ time units after $t_{d}+x+e_{\ell}$. Therefore, $\min \left(e_{h}, p_{h}-\phi_{h}\right)$ units of work due to $T_{h, c_{o}}$ does not compete with jobs in $\Psi$ before $t_{d}+x+e_{\ell}$. Hence, the competing work in $\left[t_{d}, t_{d}+x+e_{\ell}\right)$ due to the carry-out job is at most $\max \left(0, e_{h}-\left(p_{h}-\phi_{h}\right)\right)$.

Work due to jobs between $\boldsymbol{T}_{\boldsymbol{h}, \boldsymbol{c}_{\boldsymbol{i}}}$ and $\boldsymbol{T}_{\boldsymbol{h}, \boldsymbol{c}_{o}}$. The effective deadlines of successive jobs of $T_{h}$ are separated by at least $p_{h}$ time units. Therefore, the number of jobs of $T_{h}$ that lie between $\rho_{h, c_{i}}$ and $t_{d}+x+e_{\ell}-\phi_{h}$ is at most $\left\lfloor\frac{x+e_{\ell}-\xi_{h}-\phi_{h}}{p_{h}}\right\rfloor \leq$ $\frac{x+e_{\ell}-\xi_{h}-\phi_{h}}{p_{h}}$.

Combining the three components above, we have

$$
\begin{aligned}
\mathrm{D}\left(T_{h}\right) & \\
\leq & \left(\frac{x+e_{\ell}-\xi_{h}-\phi_{h}}{p_{h}}\right) \cdot e_{h}+ \\
& \max \left(0, e_{h}-\left(p_{h}-\phi_{h}\right)\right)+\min \left(e_{h}-\delta_{h}, \xi_{h}\right) \\
= & \max \left(\left(x+e_{\ell}-\xi_{h}-\phi_{h}\right) u_{h},\left(x+e_{\ell}-\xi_{h}-\phi_{h}\right) u_{h}\right. \\
& \left.+e_{h}-\left(p_{h}-\phi_{h}\right)\right)+\min \left(e_{h}-\delta_{h}, \xi_{h}\right) \\
& \left\{\text { Because } e_{h} / p_{h}=u_{h}\right\} \\
\leq & u_{h}\left(x+e_{\ell}-\xi_{h}\right)+\min \left(e_{h}-\delta_{h}, \xi_{h}\right) \\
& \left\{\text { Because } 0 \leq \phi_{h} \leq p_{h} \text { and } u_{h} \leq 1\right\} \\
= & u_{h}\left(x+e_{\ell}-\Delta_{h}-\chi_{h}\right)+\min \left(e_{h}-\delta_{h}, \Delta_{h}+\chi_{h}\right) \\
& \left\{\text { Letting } \xi_{h}=\Delta_{h}+\chi_{h} ; \text { because } d_{h, c_{i}}>t_{d}, \chi_{h}>0\right\} \\
= & \min \left(e_{h}-\delta_{h}+u_{h}\left(x+e_{\ell}-\Delta_{h}-\chi_{h}\right),\right. \\
& \left.\chi_{h}\left(1-u_{h}\right)+\Delta_{h}+u_{h}\left(x+e_{\ell}-\Delta_{h}\right)\right) \\
= & \min \left(e_{h}-\delta_{h}+u_{h}\left(x+e_{\ell}-\Delta_{h}-\chi_{h}\right),\right. \\
& \left.\left(e_{h}-\delta_{h}\right)\left(1-u_{h}\right)+\Delta_{h}+u_{h}\left(x+e_{\ell}-\Delta_{h}\right)\right) \\
& \left\{\text { Because } \chi_{h}+\Delta_{h}<e_{h}-\delta_{h} \Rightarrow \chi_{h}<e_{h}-\delta_{h}\right\} \\
\leq & \min \left(e_{h}-\delta_{h}+u_{h}\left(x+e_{\ell}-\Delta_{h}\right),\right.
\end{aligned}
$$

$$
\begin{aligned}
& \left.\left(e_{h}-\delta_{h}\right)\left(1-u_{h}\right)+\Delta_{h}+u_{h}\left(x+e_{\ell}-\Delta_{h}\right)\right) \\
\leq & \quad e_{h}-\delta_{h}+u_{h}\left(x+e_{\ell}-\Delta_{h}\right)+\min \left(0, \Delta_{h}-\left(e_{h}-\delta_{h}\right) u_{h}\right) . \\
\leq & e_{h}-\delta_{h}+u_{h}\left(x+e_{\ell}-\Delta_{h}\right)+ \\
& \min \left(0, \Delta_{h}-\left(e_{h}-\delta_{h}\right) u_{h}\right)+\max \left(0, u_{h}\left(e_{h}-e_{\ell}\right)\right) .
\end{aligned}
$$

Using Lemmas 8 and 9, a bound, as given below, on the sum of the LAG of $\Psi$ and the competing work due to tasks in $\tau_{H}$ can be obtained. This lemma is proved in [7].

Lemma $10 \mathrm{LAG}\left(\Psi, t_{d}, \mathcal{S}\right)+\sum_{T_{h} \in \tau_{H}} \mathrm{D}\left(T_{h}\right) \leq L+$ $\sum_{T_{h} \in \tau_{H}}\left(e_{h} \cdot\left(1-u_{h}\right)+u_{h} \cdot\left(x+e_{\ell}-\Delta_{h}\right)+\min \left(e_{h} \cdot u_{h}, \Delta_{h}\right)+\right.$ $\left.\max \left(0, u_{h}\left(e_{h}-e_{\ell}\right)\right)\right)$, where $L=x \cdot U_{L}+U_{H}+E_{L}$.

That completes step (S1). The next step is to determine a lower bound on the amount of such work required for tardiness of $T_{\ell, j}$ to exceed a certain amount, which we do next.

\subsection{Lower Bound on LAG + D}

Lemma 11 If (i) $\operatorname{LAG}\left(\Psi, t_{d}, \mathcal{S}\right) \leq\left(m-\left|\tau_{H}\right|\right) \cdot x+e_{\ell}$ and $\left|\tau_{H}\right|<m$ or (ii) $\operatorname{LAG}\left(\Psi, t_{d}, \mathcal{S}\right)+\sum_{T_{h} \in \tau_{H}} \mathrm{D}\left(T_{h}\right) \leq(m-$ $\left.\max \left(\left|\tau_{H}\right|-1,0\right) \cdot u_{\ell}\right) \cdot x+e_{\ell}$, then the tardiness of $T_{\ell, j}$ in $\mathcal{S}$ is at most $x+e_{\ell}$.

Proof: To prove the lemma, we show that $T_{\ell, j}$ completes executing by $t_{d}+x+e_{\ell}$. If $j>1$, then $d_{\ell, j-1} \leq t_{d}-p_{\ell}$ holds, and by $(\mathrm{P})$, we have the following.

(R) $T_{\ell, j-1}$ completes executing by $t_{d}+x+e_{\ell}-p_{\ell}$, for $j>1$.

We consider the two conditions stated in the lemma in two separate cases below. In what follows, let $H=\left|\tau_{H}\right|$. Also, without loss of generality, assume that the jobs in $\Psi$ are the only jobs of $\tau_{L}$ (or, equivalently, jobs with deadlines beyond $t_{d}$ have been discarded).

Case 1: $\operatorname{LAG}\left(\Psi, t_{d}, \mathcal{S}\right) \leq\left(m-\left|\tau_{H}\right|\right) \cdot x+e_{\ell}$ and $\left|\boldsymbol{\tau}_{\boldsymbol{H}}\right|<\boldsymbol{m}$. Let $\delta_{\ell, j}$ denote the amount of time that $T_{\ell, j}$ executed before $t_{d}$. By the conditions of this case, the amount of work pending at $t_{d}$ for jobs in $\Psi$, and hence for those of $\tau_{L}$ in $\Psi$, is at most $(m-H) \cdot x+e_{\ell}$. We first consider the case where at least $m-H$ tasks of $\tau_{L}$ are executing at any instant in $\left[t_{d}, t_{d}+x+\frac{\delta_{\ell, j}}{m-H}\right)$ and let $t_{d}+x+\frac{\delta_{\ell, j}}{m-H}+\epsilon$, where $\epsilon \geq 0$, denote the earliest instant after $t_{d}$ at which fewer than $m-H$ tasks of $\tau_{L}$ are executing. Then, the amount of work done in the interval from $t_{d}$ to $t_{d}+x+\frac{\delta_{\ell, j}}{m-H}+\epsilon$ on jobs of $\tau_{L}$ in $\Psi$ is at least $(m-H)\left(x+\epsilon+\frac{\delta_{\ell, j}}{m-H}\right)$. Therefore, the amount of work pending at $t_{d}+x+\frac{\delta_{\ell, j}}{m-H}+\epsilon$ for those jobs, and hence for $T_{\ell, j}$, is at most $(m-H) \cdot x+e_{\ell}-(m-$ $H)\left(x+\epsilon+\frac{\delta_{\ell, j}}{m-H}\right)=e_{\ell}-(m-H) \cdot \epsilon-\delta_{\ell, j}$. Hence, the latest time that $T_{\ell, j}$ completes executing is $t_{d}+x+\frac{\delta_{\ell, j}}{m-H}+$ $\epsilon+e_{\ell}-(m-H) \cdot \epsilon-\delta_{\ell, j} \leq t_{d}+x+e_{\ell}$. (Note that $T_{\ell, j}$ can execute uninterruptedly after $t_{d}+x+\frac{\delta_{\ell, j}}{m-H}+\epsilon$ until completion.) On the other hand, if fewer than $m-H$ tasks are 


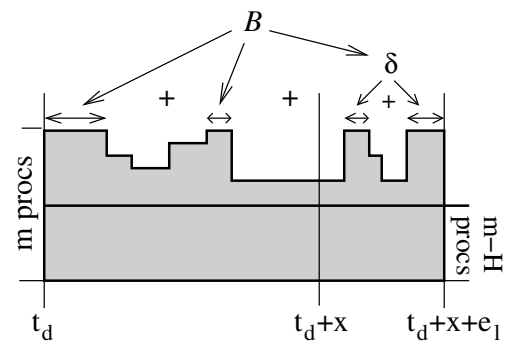

$B$ : total length of all the busy intervals in $\left[\mathrm{t}_{\mathrm{d}}, \mathrm{t}_{\mathrm{d}}+\mathrm{x}+\mathrm{e}_{1}\right)$ $\delta$ : total length of all the busy intervals in $\left[\mathrm{t}_{\mathrm{d}}+\mathrm{x}, \mathrm{t}_{\mathrm{d}}+\mathrm{x}+\mathrm{e}_{1}\right)$

Figure 4. Case 2 of Lemma 11. Sample schedule in $\left[t_{d}, t_{d}+x+\right.$ $\left.e_{\ell}\right)$.

executing at some time $t^{\prime}<t_{d}+x+\frac{\delta_{\ell, j}}{m-H}$, then fewer than $m-H$ tasks of $\tau_{L}$ have pending work at $t^{\prime}$. Because tasks of $\tau_{H}$ can execute on at most $H$ processors at any instant, $T_{\ell}$ can execute uninterruptedly from $t^{\prime}$ until $T_{\ell, j}$ completes execution. Suppose the job of $T_{\ell}$ executing at $t^{\prime}$ is $T_{\ell, j}$. Then, since $t^{\prime}<t_{d}+x+\frac{\delta_{\ell, j}}{m-H}$ holds, and the amount of work pending for $T_{\ell, j}$ is at most $e_{\ell}-\delta_{\ell, j}, T_{\ell, j}$ completes executing before $t_{d}+x+e_{\ell}$. So, assume that a prior job of $T_{\ell}$ is executing at $t^{\prime}$. In this case, $T_{\ell, j}$ could not have executed before $t_{d}$, and hence, $\delta_{\ell, j}=0$, which implies (from the definition of $t^{\prime}$ ) that $t^{\prime}<t_{d}+x$. Furthermore, $j \geq 2$ holds, and by (R), $T_{\ell, j-1}$ completes executing by $t_{d}+x$, and hence, the latest time that $T_{\ell, j}$ commences execution is at or before $t_{d}+x$, and so the latest time that $T_{\ell, j}$ completes execution is $t_{d}+x+e_{\ell}$.

Case 2: $\operatorname{LAG}\left(\Psi, t_{d}, \mathcal{S}\right)+\sum_{T_{h} \in \tau_{H}} \mathrm{D}\left(T_{h}\right) \leq\left(m-\left|\tau_{H}\right| \cdot\right.$ $\left.\boldsymbol{u}_{\ell}\right) \cdot \boldsymbol{x}+\boldsymbol{e}_{\ell}$. At the risk of some abuse in terminology, let a time interval (resp., instant) in $\left[t_{d}, t_{d}+x+e_{\ell}\right)$ in which all $m$ processors are executing a job of $\Psi$ or that part of a task in $\tau_{H}$ that can compete with $\Psi$ be referred to as a busy interval (resp., instant). Then, if pending, task $T_{\ell}$ can execute in every non-busy instant. If the latest busy instant in $\left[t_{d}, t_{d}+x+e_{\ell}\right)$ is at or before $t_{d}+x$, then because, by (R), $T_{\ell, j-1}$, if it exists, completes execution at or before $t_{d}+x$, the latest time that $T_{\ell, j}$ completes execution is $t_{d}+x+e_{\ell}$.

So, for the rest of this proof we assume that the latest busy instant is after $t_{d}+x$. Let the total lengths of the busy intervals in $\left[t_{d}+x, t_{d}+x+e_{\ell}\right)$ be $\delta \leq e_{\ell}$. (Refer to Fig. 4.) Therefore, $T_{\ell}$ can execute for at least $e_{\ell}-\delta$ time in that interval. If fewer than $m-H+1$ tasks are executing at any non-busy instant $t_{n}$ at or before $t_{d}+x$, then at most $m-H$ tasks of $\tau_{L}$ have pending work at or after $t_{n}$. Hence, since tasks in $\tau_{H}$ can execute on at most $H$ processors at any instant, $T_{\ell}$ is guaranteed uninterrupted execution from $t_{n}$ until $T_{\ell, j}$ completes. Hence, since by (R), $T_{\ell, j-1}$ (if it exists) completes execution by $t_{d}+x, T_{\ell, j}$ would complete execution no later than $t_{d}+x+e_{\ell}$. Therefore, for the rest of this case, assume the following.

(N) At least $\min (m-H+1, m)$ tasks are executing at every non-busy instant in $\left[t_{d}, t_{d}+x\right)$.

Let $B$ denote the total length of all the busy intervals in $\left[t_{d}, t_{d}+x+e_{\ell}\right)$. (Refer to Fig. 4.) If $B \leq x-x \cdot u_{\ell}$, then $T_{\ell}$ can execute for at least $x \cdot u_{\ell}+e_{\ell}$ time in $\left[t_{d}, t_{d}+x+e_{\ell}\right)$. By Lemma $4, \operatorname{lag}\left(T_{\ell}, t_{d}, \mathcal{S}\right) \leq x \cdot u_{\ell}+e_{\ell}$, and hence, $T_{\ell, j}$ would complete executing at or before $t_{d}+x+e_{\ell}$. So, assume $B=x-x \cdot u_{\ell}+\delta_{1}$, where $\delta_{1}>0$. With this assumption, we now compute the total amount of work done in $\left[t_{d}, t_{d}+\right.$ $\left.x+e_{\ell}\right)$. The total amount of work done in all busy intervals in $\left[t_{d}, t_{d}+x+e_{\ell}\right)$ is $m \cdot B$. By $(\mathrm{N})$, at least $\min (m-H+1, m)$ tasks are executing at every non-busy instant in $\left[t_{d}, t_{d}+x\right)$. The total length of all the non-busy intervals in $\left[t_{d}, t_{d}+x\right)$ is $x-(B-\delta)$. Therefore, the amount of work done in all the non-busy intervals in $\left[t_{d}, t_{d}+x\right)$ is at least $\min (m-H+$ $1, m) \cdot(x-B+\delta)$. The total length of all the non-busy intervals in $\left[t_{d}+x, t_{d}+x+e_{\ell}\right)$ is $e_{\ell}-\delta$, and at least task $T_{\ell}$ of $\tau_{L}$ has pending jobs in $\Psi$ until $t_{d}+x+e_{\ell}$, and hence, executes in every non-busy instant in $\left[t_{d}+x, t_{d}+x+e_{\ell}\right)$. (Otherwise, it would imply that $T_{\ell, j}$ has completed executing before $t_{d}+x+e_{\ell}$, completing the proof, as well). Hence, the total amount of work done in $\left[t_{d}, t_{d}+x+e_{\ell}\right)$ is at least $m B+\min (m-H+1, m) \cdot(x-B+\delta)+\left(e_{\ell}-\delta\right)$, which, on substituting $x-x \cdot u_{\ell}+\delta_{1}$ for $B$, simplifies to $m x-H$. $x \cdot u_{\ell}+(m-H) \cdot \delta+x \cdot u_{\ell}+H \cdot \delta_{1}+\left(e_{\ell}-\delta_{1}\right)$, for $H>0$ and $m \cdot(x+\delta)+e_{\ell}-\delta$, for $H=0$.

By the condition of this case, the amount of work that needs to be done in $\left[t_{d}, t_{d}+x+e_{\ell}\right)$ for jobs in $\Psi$ and of tasks in $\tau_{H}$ that can compete with $\Psi$ is at most $m x-\max (H-1,0) \cdot x \cdot$ $u_{\ell}+e_{\ell}$. Therefore, the amount of work pending at $t_{d}+x+e_{\ell}$ is at most $-(H-1) \cdot \delta_{1}-(m-H) \cdot \delta$, for $1 \leq H \leq m$, and is at most $-(m-1) \cdot \delta$, for $H=0$. Because $\delta$ and $\delta_{1}$ are positive, both the above bounds are negative. Thus, no work of jobs in $\Psi$, and in particular, that of $T_{\ell, j}$, can be pending at $t_{d}+x+e_{\ell}$.

This completes step (S2). We are left with determining a value for $x$ for which the tardiness of $T_{\ell, j}$ is at most $x+e_{\ell}$.

\subsection{Finishing Up}

Solving for $x$ using Lemma 8 and the first condition in Lemma 11, i.e., solving for $x$ in $x \cdot U_{L}+U_{H}+E_{L}+E_{H} \leq$ $\left(m-\left|\tau_{H}\right|\right) x+e_{\ell}$, yields

$$
x \geq \frac{E_{L}+U_{H}+E_{H}-e_{\ell}}{\left(m-\left|\tau_{H}\right|\right)-U_{L}},
$$

where $E_{H}$ is as in Fig. 2. Solving using Lemma 10 and the second condition of Lemma 11, i.e., using $\sum_{T_{h} \in \tau_{H}}\left(e_{h} \cdot(1-\right.$ $\left.u_{h}\right)+u_{h} \cdot\left(x+e_{\ell}-\Delta_{h}\right)+\min \left(e_{h} \cdot u_{h}, \Delta_{h}\right)+\max \left(0, u_{h} \cdot\left(e_{h}-\right.\right.$ $\left.\left.e_{\ell}\right)\right)+x \cdot U_{L}+U_{H}+E_{L} \leq m x-\max \left(\left|\tau_{H}\right|-1,0\right) \cdot x \cdot u_{\ell}+e_{\ell}$, yields

$$
x \geq \frac{E_{L}+U_{H}+E_{H}^{\prime}-e_{\ell}}{m-\max \left(\left|\tau_{H}\right|-1,0\right) \cdot u_{\ell}-U_{L}-U_{H}^{\prime}},
$$

where $E_{H}^{\prime}$ and $U_{H}^{\prime}$ are as in Fig. 2. Hence, if $x$ is smaller of the two values that are on the right-hand sides of (14) and (15), then the tardiness of $T_{\ell, j}$ would not exceed $x+e_{\ell}$. A value of $x$ that is independent of the parameters of $T_{\ell}$ is obtained by replacing $e_{\ell}$ by $e_{\min }$ and $u_{\ell}$ by $u_{\max }\left(\tau_{L}\right)$ in (14) 


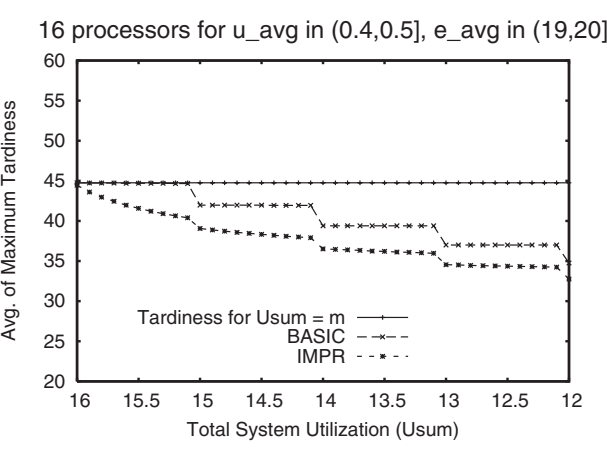

(a)

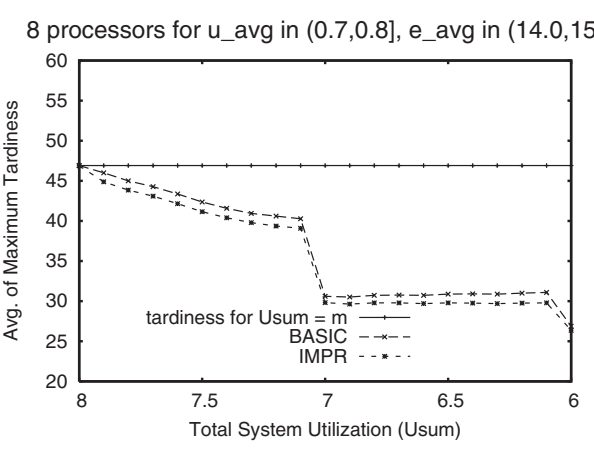

(b)

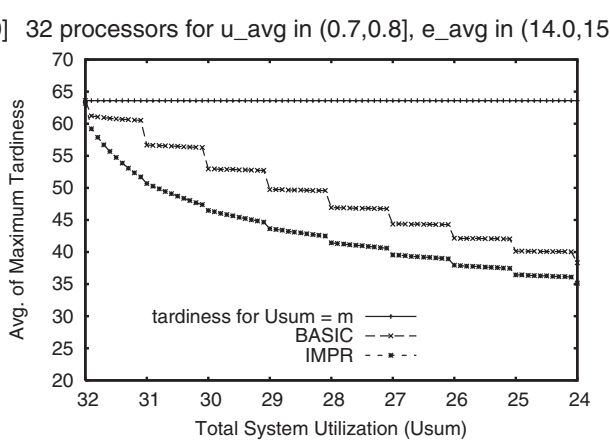

(c)

Figure 5. Tardiness by $U_{\text {sum }}$ using the BASIC and IMPR bounds for (a) $m=16,0.4<u_{\text {avg }} \leq 0.5$, and $19.0<e_{\text {avg }} \leq 20.0$, (b) $m=8$, $0.7<u_{\text {avg }} \leq 0.8$, and $14.0<e_{\text {avg }} \leq 15.0$, and (c) $m=32,0.7<u_{\text {avg }} \leq 0.8$, and $14.0<e_{\text {avg }} \leq 15.0$.

and (15). Similarly, the $e_{\ell}$ term in the expression for $E_{H}^{\prime}$ has to be replaced by $e_{\max }\left(\tau_{L}\right)$. By inducting over the jobs of $\tau_{L}$ in the non-decreasing order of their deadlines, we have the following.

Theorem 1 EDF-hl ensures a tardiness of at most $\min \left(X_{1}, X_{2}\right)+e_{k}$ to every task $T_{k}$ of $\tau_{L}$ if $\left|\tau_{H}\right| \leq m$ and $U_{\text {sum }}(\tau) \leq m$, where $X_{1}=\frac{E_{L}+U_{H}+E_{H}-e_{\min }\left(\tau_{L}\right)}{\left(m-\left|\tau_{H}\right|\right)-U_{L}}$ and $X_{2}=\frac{E_{L}+U_{H}+E_{H}^{\prime}-e_{\min }\left(\tau_{L}\right)}{m-\max \left(\left|\tau_{H}\right|-1,0\right) \cdot u_{\max }\left(\tau_{L}\right)-U_{L}-U_{H}^{\prime}}$.

Conditions for bounded tardiness. Since the derivation was based on the assumption that $x \geq 0, X_{1}$ and $X_{2}$ are valid only if their denominators are non-negative. $X_{1}$ and $X_{2}$ are bounded only if their denominators are greater than zero. Hence, if the sum of the utilizations of the $\Lambda-1$ heaviest tasks in $\tau_{L}$ is less than $m-\left|\tau_{H}\right|$, then $X_{1}$ is bounded. Similarly, $X_{2}$ is bounded only if the sum of the utilizations of the heaviest $\Lambda-1$ tasks in $\tau_{L}$ is less than $m-\max \left(\left|\tau_{H}\right|-\right.$ $1,0) \cdot u_{\max }\left(\tau_{L}\right)-U_{H}^{\prime}$. Hence, if either of the above conditions holds, then bounded tardiness is guaranteed to tasks in $\tau_{L}$.

\section{Performance Evaluation}

In this section, we present the results of a simulation-based evaluation of (i) the tardiness-utilization trade-off possible in the absence of privileged tasks and (ii) the range of the tardiness bound guaranteed by EDF-hl on an average. Due to space constraints, only a subset of the results is presented here.

Tardiness-utilization trade-off. As mentioned earlier, EDF-hl reduces to EDF in the absence of privileged tasks. Hence, in this case, the tardiness bound given in Thm. 1 applies to every task in $\tau$. Note that the tardiness bound is expressed in terms of $U_{\text {sum }}(\tau)$ in addition to individual task parameters. Hence, an alternative to EDF-hl for guaranteeing lower tardiness is to lower $U_{\text {sum }}$. This approach may be preferable if a majority of the tasks require lower tardiness and the gains are reasonable for slight decreases in $U_{\text {sum }}$.

In the absence of privileged tasks, using a slightly different, but more complicated, analysis than that used in Sec. 3 or in
[8], it can be shown that

$$
U_{L} \leq \sum_{k=1}^{\Lambda-1} \frac{u_{k}^{2}(m-\Lambda)}{\left(m-U_{\text {sum }}\right)+u_{k}\left(U_{\text {sum }}-\Lambda\right)},
$$

which when used in the expression for the tardiness bound in Thm. 1 results in slightly lower values. We will refer to the bound given in Thm. 1 as BASIC and the bound obtained by using (16) as IMPR.

We evaluated the tardiness-utilization trade-off that is possible by generating random task sets with varying values for $U_{\text {sum }}$ and computing the BASIC and IMPR bounds for each and comparing these bounds with those obtained from our earlier work, when $U_{\text {sum }}=m$ [8]. Simulation experiments were conducted for four, eight, 16, and 32 processors, with $U_{\text {sum }}$ varying between $3 \mathrm{~m} / 4$ and $m$ in increments of 0.1 . 600,000 task sets, with at least $m+1$ tasks in each, were generated for each $\left(U_{\text {sum }}, m\right)$ pair. The maximum utilization of any task in a task set varied uniformly from 0.5 to 1.0. The task sets generated were grouped based on $u_{\text {avg }}$ and $e_{\text {avg }}$, where $u_{\text {avg }}$ and $e_{\text {avg }}$ are the averages of the highest $\left\lfloor U_{\text {sum }}\right\rfloor$ task utilizations and execution costs, respectively. The variation in tardiness (mean of the maximum tardiness for all task sets in a group) with $U_{\text {sum }}$ for $m=16$, when $0.4<u_{\text {avg }} \leq 0.5$ and $19.0<e_{\text {avg }} \leq 20.0$, and $m=8$ and $m=32$, when $0.7<u_{\text {avg }} \leq 0.8$ and $14.0<e_{\text {avg }} \leq 15.0$, are presented in Fig. 5. Note that the rate at which tardiness drops with decreasing $U_{\text {sum }}$ is higher when $u_{\text {avg }}$ is higher (in the $(0.7,0.8]$ range). Furthermore, the rate at which tardiness drops with $U_{\text {sum }}$ decreases with decreasing $U_{\text {sum }}$. For instance, in inset (c), reducing $U_{\text {sum }}$ to 31.0 (which is $96.8 \%$ of $m(=32)$ ) lowers tardiness to less than 50.0 from over 60.0, which is a drop of over $20 \%$, whereas to lower tardiness to less than $40.0, U_{\text {sum }}$ has to be decreased to approximately 27.0 (which is $84.3 \%$ of $m$ ). Hence, setting $U_{\text {sum }}$ to a value slightly lower than $m$ may be appropriate when high utilization tasks are present in the task system. At this point, we would like to note that these characteristics should be attributed to the bounds derived (and to the analysis) and not to the algorithm per se.

Tardiness bounds for EDF-hl. We also evaluated the tardiness bounds that can be guaranteed to unprivileged tasks on an average under EDF-hl for $m=4$ and $m=16$, with 


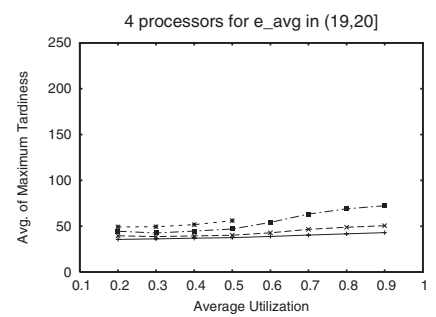

(a)

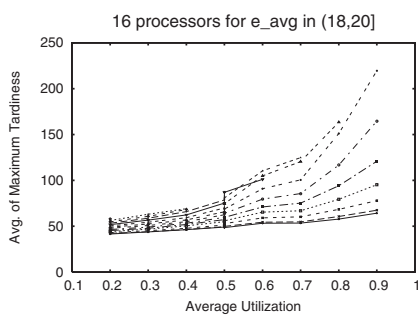

(b)

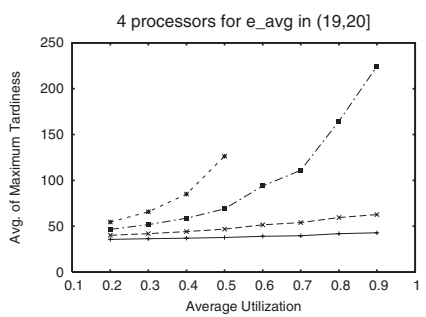

(c)

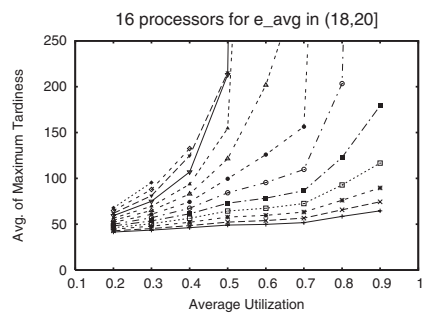

(d)

Figure 6. Experimental evaluation of the tardiness bounds guaranteed by Thm. 1 under EDF-hl for tasks in $\left|\tau_{L}\right|$. LU with (a) $m=4$ and (b) $m=16$. HU with (c) $m=4$ and (d) $m=16$. The different curves in each inset correspond to different values of $\left|\tau_{H}\right|$. $\left|\tau_{H}\right|=0$ for the bottom-most curve and is greater by one for each curve higher up.

$U_{\text {sum }}=m$. The task sets generated were grouped based on the average of the $m$ highest task utilizations and the utilizations of the tasks in $\tau_{H}$, denoted $u_{a v g}$. ( $e_{a v g}$ is with respect to execution costs, analogously.) For each task set generated, the number of tasks in $\tau_{H}$ was varied from zero to $m$, and for each $\left|\tau_{H}\right|$, the members of $\tau_{H}$ were chosen in two different ways: first, as tasks with the lowest $\left|\tau_{H}\right|$ utilizations in the generated task set (denoted LU), and then, as tasks with the highest $\left|\tau_{H}\right|$ utilizations (denoted HU). The variation in tardiness with $u_{\text {avg }}$ as the number of privileged tasks is increased is plotted in Fig. 6 for both LU and HU. As expected, tardiness increases with $\left|\tau_{H}\right|$ and $u_{a v g}$, and the increase is higher for $\mathrm{HU}$ than for LU. The tardiness bounds computed grew to unbounded values for certain task sets at high values of $\left|\tau_{H}\right|$, with the percentage of such task sets increasing with increasing $u_{\text {avg. }}$. Further details on such task sets can be found in [7].

\section{Conclusion}

We have addressed the issue of supporting tasks whose tolerance to tardiness is lower than that currently known to be possible under EDF. We have proposed a new scheduling policy called EDF-hl, which is based on EDF, and have shown that under EDF-hl, a limited number of privileged tasks can be guaranteed any tardiness, including zero tardiness, and that bounded tardiness can be guaranteed to the remaining tasks if their utilizations are restricted. The tardiness bound derived is a function of $U_{\text {sum }}$, in addition to individual task parameters, and hence, tardiness for all tasks can be lowered by slightly lowering $U_{\text {sum. }}$. We have, through simulations, assessed the impact of privileged tasks on the tardiness bounds that can be guaranteed to the remaining tasks, and the tardiness-utilization trade-off that is possible in the absence of privileged tasks.

This problem of supporting sporadic tasks with different tardiness requirements may alternatively be viewed as one of supporting tasks with relative deadlines at least periods. The EDF schedulability tests available for task systems with relative deadlines equal to periods on a multiprocessor, though applicable when deadlines may exceed periods also, are pessimistic and tend to under-utililize the underlying platform. The work presented in this paper is an attempt towards remedying this limitation.

\section{References}

[1] J. Anderson, V. Bud, and U. Devi. An EDF-based scheduling algorithm for multiprocessor soft real-time systems. In Proc. of the 17th Euromicro Conf. on Real-Time Systems, pages 199-208, July 2005.

[2] B. Andersson and J. Jonsson. The utilization bounds of partitioned and pfair static-priority scheduling on multiprocessors are 50\%. In Proc. of the 15th Euromicro Conf. on Real-Time Systems, pages 33-40, July 2003.

[3] S. Baruah. Optimal utilization bounds for the fixed-priority scheduling of periodic task systems on identical multiprocessors. IEEE Trans. on Computers, 53(6):781-784, 2004.

[4] S. Baruah, N. Cohen, C.G. Plaxton, and D. Varvel. Proportionate progress: A notion of fairness in resource allocation. Algorithmica, 15:600-625, 1996

[5] S. Cho, S.K. Lee, S. Ahn, and K.J. Lin. Efficient real-time scheduling algorithms for multiprocessors. IEICE Trans. on Communications, E85-B(12):2859-2867, December 2002.

[6] M. Dertouzos. Control robotics: The procedural control of physical processes. In Proc. of IFIP Cong., pages 807-813, 1974.

[7] U. Devi and J. Anderson. Flexible tardiness bounds for sporadic realtime task systems on multiprocessors (extended version). Available at http://www.cs.unc.edu/ ${ }^{\sim}$ anderson/papers.html, October 2005.

[8] U. Devi and J. Anderson. Tardiness bounds for global EDF scheduling on a multiprocessor. In Proc. of the 26th IEEE Real-Time Systems Symp., pages 330-341, December 2005.

[9] J. Carpenter et al. A categorization of real-time multiprocessor scheduling problems and algorithms. In Joseph Y. Leung, editor, Handbook on Scheduling Algorithms, Methods, and Models, pages 30.1-30.19. Chapman Hall/CRC, Boca Raton, Florida, 2004.

[10] C.L. Liu and J.W. Layland. Scheduling algorithms for multiprogramming in a hard-real-time environment. Journal of the Association for Computing Machinery, 20(1):46-61, 1973.

[11] J.M. Lopez, M. Garcia, J.L. Diaz, and D.F. Garcia. Worst-case utilization bound for EDF scheduling on real-time multiprocessor systems. In Proc. of the 12th Euromicro Conf. on Real-Time Systems, pages 25-34, June 2000.

[12] A. Srinivasan and J. Anderson. Efficient scheduling of soft real-time applications on multiprocessors. In Proc. of the 15th Euromicro Conf. on Real-Time Systems, pages 51-59, July 2003.

[13] P. Valente and G. Lipari. An upper bound to the lateness of soft realtime tasks scheduled by EDF on multiprocessors. In Proc. of the 26th IEEE Real-Time Systems Symp., pages 311-320, December 2005. 